\title{
Coeficiente $K p$ del tanque evaporímetro clase "A" para la estimación de la evapotranspiración de referencia
}

\author{
Coefficient Kp of class "A" pan for the estimation of reference evapotranspiration \\ Juan Alejandro Villazón Gómez ${ }^{*}$, Pavel Noris Noris², Ranses J. Vázquez Montenegro ${ }^{3}$, \\ George Martín Gutiérrez", Yakelín Cobo Vidal ${ }^{4}$
}

\begin{abstract}
RESUMEN
La determinación de la evapotranspiración de referencia resulta de suma importancia para un adecuado manejo del riego. Esta puede ser determinada por diferentes métodos, entre los cuales se encuentra el del tanque evaporímetro clase "A", que utiliza el coeficiente $K p$ para corregir las disimilitudes entre la evaporación desde una superficie libre de agua y dicha evapotranspiración de referencia. El trabajo se desarrolló con el objetivo de calibrar el coeficiente $K p$ del tanque evaporímetro clase "A" para el cálculo de la evapotranspiración de referencia decenal en áreas agropecuarias del municipio de Mayarí en la provincia de Holguín. Se utilizaron los datos observados en dos estaciones meteorológicas (Guaro y Pinares de Mayarí) entre 1975-2015. Con los años pares dentro de la serie temporal se calibró el coeficiente $K p$, a partir de la ecuación del tanque evaporímetro; con los años impares se validó la ETo obtenida por dicha ecuación comparándola, mediante un análisis de regresión simple y los índices de concordancia (d) y confianza (c) con la ETo FAO Penman-Monteith. Los modelos de regresión con mejores coeficientes se obtuvieron con la ETo estimada_1 con los coeficientes $K p$ calibrados a partir de la ecuación del tanque evaporímetro clase "A". Esta mostró índices de concordancia más cercanos a 1. Los índices de confianza de esta ETo se clasificaron como bueno y medio, por lo que constituye un método óptimo, rápido y sencillo a utilizar como herramienta para el manejo del riego.
\end{abstract}

Palabras clave: Índices estadísticos; Manejo del riego; Tanque evaporímetro clase "A".

\begin{abstract}
The determination of the reference evapotranspiration is of utmost importance for an adequate irrigation management. This can be determined by different methods, among which is that of the Class "A" pan, which uses the Kp coefficient to correct the dissimilarities between evaporation from a water-free surface and said reference evapotranspiration. The work was developed with the objective of calibrating the Kp coefficient of the Class " $A$ " pan for the calculation of the decennial reference evapotranspiration in agricultural areas of the municipality of Mayari in the province of Holguin. The data observed in two meteorological stations (Guaro and Pinares de Mayari) between 1975-2015 were used. With the even years within the time series, the Kp coefficient was calibrated, from the equation of the Class "A" pan; with odd years the ETo obtained by said equation was validated by comparing $i$, by means of a simple regression analysis and the concordance $(d)$ and confidence $(c)$ indexes with the FAO Penman-Monteith ETo. The regression models with the best coefficients were obtained with the estimated_l ETo with the Kp coefficients calibrated from the Class "A" pan equation. This showed concordance indexes closer to 1. The confidence indexes of this ETo were classified as good and intermediate, which is why it is an optimal, fast and simple method to be used as a tool for irrigation management.

Keywords: Statisticals index; Irrigation management; Class "A” pan.
\end{abstract}

\section{Introducción}

La ecuación de FAO Penman-Monteith es considerada el método estándar para la estimación de la evapotranspiración de referencia (Allen et al., 2006; Martins et al., 2014). Aunque ha demostrado un comportamiento adecuado de las predicciones en las regiones y condiciones climáticas más variadas

\footnotetext{
1 Universidad de Holguín. Sede José de la Luz y Caballero, Facultad de Ciencias Agropecuarias, Centro de Estudios para Agroecosistemas Áridos (CEAAR), Holguín, Cuba.

2 Centro Provincial de Meteorología, Delegación Provincial del Ministerio de Ciencias, Tecnología y Medio Ambiente (CITMA), Holguín, Cuba.

3 Instituto de Meteorología, Ministerio de Ciencias, Tecnología y Medio Ambiente (CITMA), La Habana, Cuba.

4 Estación Provincial de Investigaciones de la Caña de Azúcar (EPICA), Subdirección de I+D, Guaro s/n, Mayarí, Holguín, Cuba.

* Autor por correspondencia: villazon@uho.edu.cu.
} 
(Puppo y García, 2009) tiene el inconveniente de necesitar una gran cantidad de datos climáticos no siempre disponibles (Sánchez, 2001).

Desde la década de 1970, se generalizó el uso del tanque evaporímetro clase "A" para determinar el momento de riego de diferentes cultivos, al ser la base para el pronóstico de riego por el método bioclimático (Bonet et al., 2012). El método del tanque evaporímetro clase "A" tiene como ventaja su simplicidad, al medir los efectos integrados del clima a partir de una única medida: la evaporación de una superficie libre de agua. De la misma forma, los bajos costos de instalación y mantenimiento han propiciado su amplia utilización en proyectos y programaciones de riego (Sentelhas y Folegatti, 2003).

La evapotranspiración en las áreas cultivadas ocurre bajo el efecto de los mismos factores climáticos que propician la evaporación desde la superficie del tanque, pero una serie de elementos provocan diferencias, las cuales quedan corregidas al utilizar el coeficiente $K p$ (Puppo y García, 2009). Aunque este coeficiente, puede obtenerse con una ecuación indirecta empírica, es recomendable calibrar $K p$ a partir de la evapotranspiración obtenida por lisímetros o por la ecuación de FAO Penman-Monteith (Allen et al., 2006).

Por lo antes expuesto, el objetivo de este trabajo es calibrar el coeficiente $K p$ del tanque evaporímetro clase "A" para el cálculo de la evapotranspiración de referencia decenal en áreas agropecuarias del municipio de Mayarí en la provincia de Holguín.

\section{Materiales y métodos}

El trabajo se desarrolló con los datos tomados, en el período 1990-2015, en dos estaciones meteorológicas de la provincia de Holguín: Guaro $\left(20^{\circ} 40^{\prime} 21^{\prime \prime} \mathrm{N}\right.$ y $\left.75^{\circ} 46^{\prime} 57^{\prime \prime} \mathrm{W}\right)$, con una precipitación de $1099,3 \mathrm{~mm}$ al año $25,6^{\circ} \mathrm{C}$ de temperatura media, a $21 \mathrm{msnmm}$ (metros sobre el nivel medio del mar) y Pinares de Mayarí (202 $29^{\prime} 14^{\prime \prime} \mathrm{N}$ y $75^{\circ} 47^{\prime} 26^{\prime}$ " W), $1871,0 \mathrm{~mm}$ de lluvia anuales, una temperatura media de $21,6^{\circ} \mathrm{C}$, a $647 \mathrm{msnmm}$.

Se revisaron las bases de datos de las estaciones meteorológicas para encontrar datos faltantes y se realizó un análisis de correlación entre ambas para completar los datos faltantes mediante regresión. Los años comprendidos en el período estudiado (26 años) se dividieron en dos grupos de 13 años cada uno. Con los años pares se calibró el coeficiente $K p$.
La evaporación del tanque evaporímetro clase "A" está relacionada con la evapotranspiración de referencia por un coeficiente empírico derivado del mismo tanque:

$$
\mathrm{ET}_{0}=\mathrm{K}_{\mathrm{p}} * \mathrm{E}_{\mathrm{pan}}
$$

Donde ETo es la evapotranspiración de referencia $\left(\mathrm{mm} \mathrm{día}^{-1}\right) ; \mathrm{K} p$ es el coeficiente del tanque evaporímetro clase "A" (adimensional) y Epan es la evaporación del tanque evaporímetro (mm día $\left.{ }^{-1}\right)$.

A partir de (1) se calcula el coeficiente del tanque evaporímetro clase "A":

$$
\mathrm{K}_{\mathrm{p}}=\frac{\mathrm{ET}_{0}}{\mathrm{E}_{\mathrm{pan}}}
$$

Al mismo tiempo se determinó el coeficiente $K p$ calculado por la ecuación propuesta por Allen et al. (2006):

$$
\begin{gathered}
\mathrm{Kp}=0,108-0,0286 \mathrm{u}_{2}+0.0422 \ln (\text { fet })+ \\
0,1434 \ln \left(\mathrm{HR}_{\mathrm{m}}\right)-0,000631[\ln (\mathrm{fet})]^{2} \ln \left(\mathrm{HR}_{\mathrm{M}}\right)
\end{gathered}
$$

Donde $u_{2}$ es la velocidad del viento a $2 \mathrm{~m}$ de altura $\left(\mathrm{m} \mathrm{s}^{-2}\right)$; fet es el borde o distancia al borde de la superficie identificada, fijada en $1.000 \mathrm{~m}$, que es la distancia máxima permitida para la estimación del coeficiente $K p$ (Allen et al., 2006) y $H R m$ es la humedad relativa media (\%).

La velocidad del viento $u_{2}$ se mide a una altura estándar elegida para todas las estaciones meteorológicas $(10 \mathrm{~m})$, pero para calcular la evapotranspiración se necesita dicha variable climática medida a $2 \mathrm{~m}$ de altura; por lo que para ajustar los datos medidos se utilizó la relación logarítmica:

$$
\mathrm{u}_{2}=\mathrm{u}_{\mathrm{z}} \frac{4,87}{\ln (67,8 \mathrm{z}-5,42)}
$$

Donde $u_{z}$ es la velocidad del viento medida a z $\mathrm{m}$ sobre la superficie $\left(\mathrm{m} \mathrm{s}^{-2}\right)$ y $z$ es la altura de medición sobre la superficie (10 $\mathrm{m}$ en ambas estaciones).

Los valores del coeficiente $K p$ obtenidos por la metodología de Allen et al. (2006) fueron comparados con los obtenidos a partir de la ecuación del tanque evaporímetro clase "A" para verificar si se adapta a las condiciones ambientales locales.

La evapotranspiración de referencia se estimó a partir de la ecuación FAO Penman-Monteith, propuesta por Allen et al. (2006): 


$$
\mathrm{ET}_{0}=\frac{0,408 \Delta\left(\mathrm{R}_{\mathrm{n}}+\mathrm{G}\right)+\gamma \frac{900}{\mathrm{~T}+273} \mathrm{u}_{2}\left(\mathrm{e}_{\mathrm{s}}-\mathrm{e}_{\mathrm{a}}\right)}{\Delta+\gamma\left(1+0,34 \mathrm{u}_{2}\right)}
$$

Donde ETo es la evapotranspiración de referencia $\left(\mathrm{mm} \mathrm{día}^{-1}\right) ; \Delta$ es la pendiente de la curva de presión de vapor $\left(\mathrm{kPa}^{\circ} \mathrm{C}^{-1}\right) ; R n$ es la radiación neta en la superficie del cultivo ( $\mathrm{MJ} \mathrm{m}^{-2}$ día $^{-1}$ ); $G$ es el flujo de calor del suelo, $\gamma$ es la constante psicrométrica $\left(\mathrm{kPa}^{\circ} \mathrm{C}^{-1}\right) ; T$ es la temperatura media del aire a $2 \mathrm{~m}$ de altura $\left({ }^{\circ} \mathrm{C}^{-1}\right)$ y $u_{2}$ es la velocidad del viento a $2 \mathrm{~m}$ de altura $\left(\mathrm{m} \mathrm{s}^{-2}\right)$.

Con los años impares del período se validó la ETo estimada con los coeficientes $K p$ determinados a partir de la ecuación del tanque evaporímetro clase "A" (ETo estimada_1) y de la ecuación propuesta por Allen et al. (2006) (ETo estimada_2). Se realizó un análisis de regresión simple y se determinaron los índices de concordancia y de confianza o desempeño.

Índice de concordancia (Index of agreement, $d$ ) (Wilmott, 1985):

$\mathrm{d}=1\left[\frac{\sum_{\mathrm{i}=1}^{\mathrm{n}}\left(\mathrm{ETi}_{\mathrm{e}}-\mathrm{ETi}_{\mathrm{m}}\right)^{2}}{\sum_{\mathrm{i}=1}^{\mathrm{n}}\left(\left|\mathrm{ETi}_{\mathrm{e}}-\mathrm{ETmean}_{\mathrm{m}}\right|+\left|\mathrm{ETi}_{\mathrm{m}}-\mathrm{ETmean}_{\mathrm{m}}\right|\right)^{2}}\right]$

Donde $n$ es el número de pares de datos, $E T i_{e}$ es la i-enésima observación de la evapotranspiración estimada por el modelo a comparar, $E T i_{m}$ es la i-enésima observación de la evapotranspiración estimada por el método de FAO Penman-Monteith y ETmean ${ }_{m}$ es la media de la evapotranspiración estimada por el método de FAO Penman-Monteith. Es una medida estandarizada del grado de error de la predicción del modelo, en un rango desde 0 hasta
1. Cuando $d=1$, existe una concordancia perfecta entre los valores determinados experimentalmente y los estimados por el modelo; en sentido contrario, cuando $d=0$, nos indica falta de concordancia entre las variables.

Coeficiente de confianza o desempeño (c) (Camargo y Sentelhas, 1997):

$$
\mathrm{c}=\mathrm{d} * \mathrm{r}
$$

Donde $d$ es el índice de concordancia y $r$ es el coeficiente de correlación. Los valores de $c$ fueron clasificados de acuerdo a las categorías propuestas por Camargo y Sentelhas (1997).

\section{Resultados y discusión}

La Tabla 1 muestra el promedio del coeficiente $K p$ determinado decenalmente a partir de la ecuación del tanque evaporímetro clase "A" aplicada a los años pares de la serie temporal estudiada. Los resultados obtenidos fueron superiores a los observados por Bonet et al. (2012), quienes en el cultivo de la piña en la provincia de Ciego de Ávila encontraron valores del coeficiente $K p$ de 0,58 y 0,65 para los períodos seco y lluvioso, respectivamente.

Los valores del coeficiente $K p$ son, generalmente, más cercanos a 1 en Pinares de Mayarí que en Guaro; lo cual indica que existen menos diferencias entre los tenores de la evapotranspiración de referencia y la evaporación del tanque evaporímetro en la primera estación meteorológica mencionada.

La Figura 1 muestra las regresiones de las ETo estimadas 1 y 2 con respecto a la evapotranspiración de referencia obtenida a partir de la ecuación de FAO Penman-Monteith. En ambas estaciones

Tabla 1. Coeficientes $K p$ determinados a partir de la ecuación del tanque evaporímetro clase "A".

\begin{tabular}{lcccccccccccc}
\hline Decenas & ene & feb & mar & abr & may & jun & jul & ago & sep & oct & nov & dic \\
\hline Primera & 0,66 & 0,66 & 0,64 & 0,65 & 0,67 & 0,70 & 0,68 & 0,67 & 0,68 & 0,71 & 0,71 & 0,70 \\
Segunda & 0,65 & 0,66 & 0,68 & 0,68 & 0,66 & 0,71 & 0,68 & 0,67 & 0,69 & 0,70 & 0,67 & 0,67 \\
Tercera & 0,63 & 0,65 & 0,67 & 0,73 & 0,70 & 0,69 & 0,66 & 0,65 & 0,67 & 0,72 & 0,65 & 0,63 \\
\hline \multicolumn{10}{c}{ Pinares de Mayarí } \\
\hline Primera & 0,69 & 0,71 & 0,72 & 0,79 & 0,83 & 0,85 & 0,79 & 0,82 & 0,79 & 0,75 & 0,70 & 0,68 \\
Segunda & 0,65 & 0,75 & 0,72 & 0,75 & 0,77 & 0,82 & 0,82 & 0,82 & 0,84 & 0,79 & 0,72 & 0,71 \\
Tercera & 0,67 & 0,74 & 0,73 & 0,84 & 0,83 & 0,82 & 0,77 & 0,76 & 0,84 & 0,75 & 0,71 & 0,69 \\
\hline
\end{tabular}



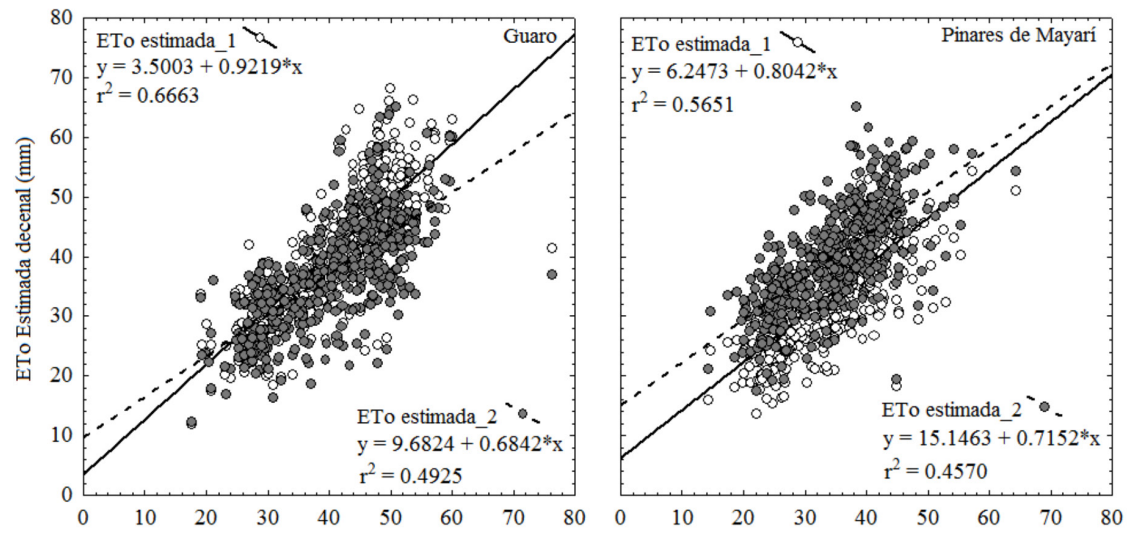

Figura 1. Ecuaciones de regresión a partir de la evapotranspiración de referencia estimada por el método de FAO Penman-Monteith (regresora) y la ETo estimada a partir de los coeficientes Kp (dependientes).

meteorológicas los valores estimados a partir del coeficiente $K p$ calibrado (ETo estimada_1) mostró con los coeficientes $K p$ obtenidos

En el modelo de regresión lineal simple puede observarse que el parámetro que representa la pendiente de la recta $(b)$ se encuentra en todos los casos por debajo de 1 , lo que indica una tendencia a subestimar, por parte de las ETo estimadas 1 y 2 , los valores de evapotranspiración de referencia obtenidos por la ecuación patrón. No obstante, resulta válido señalar la mayor cercanía a la unidad, en el modelo de regresión de la ETo estimada_1 para la estación meteorológica de Guaro, del parámetro $b$. Lo que revela una mayor semejanza de los valores de la ETo estimada_1 con la evapotranspiración de referencia por el modelo FAO Penman-Monteith.

El comportamiento de la ETo estimada_2 durante el análisis de regresión difiere de lo observado por Andrade et al. (2016). Al estimar la evapotranspiración de referencia con la ecuación del tanque evaporímetro clase "A" mediante la utilización del coeficiente $K p$ a partir de la ecuación propuesta por Allen et al. (2006), con los datos medidos durante un periodo de 10 años en la estación meteorológica principal del municipio de Lavras (estado de Minas Gerais,
Brasil), y compararla con la ETo por el método FAO Penman-Monteith encontraron un coeficiente de regresión de 0,9069.

De la misma forma, en la región medio-norte del estado de Mato Grosso (Brasil), Souza et al. (2015) al comparar la ETo obtenida a partir de la ecuación del tanque evaporímetro clase "A", mediante la utilización de coeficiente $K p$ (Allen et al., 2006), con la ETo de la ecuación FAO Penman-Monteith encontraron un coeficiente de regresión de 0,6226.

La Tabla 2 muestra el comportamiento de los índices utilizados para validar la evapotranspiración de referencia (ETo estimada_1 y ETo estimada_2) obtenida a partir del coeficiente $K p$. Con respecto al índice de concordancia se aprecia que los valores más cercanos a 1 se encontraron en la ETo estimada_1 para las estaciones meteorológicas de Guaro y Pinares de Mayarí. En el caso de la ETo estimada_2 se determinó un menor índice de concordancia. Para las dos ETo estimadas, los mejores resultados se observaron en la estación meteorológica de Guaro.

En cuanto al índice de confianza, el mismo se clasificó para la ETo estimada_1 como bueno y medio en las estaciones meteorológicas de Guaro

Tabla 2. Índices de concordancia y desempeño para las ETo estimadas en Guaro y Pinares de Mayarí.

\begin{tabular}{lcccc}
\hline \multirow{2}{*}{ Estaciones meteorológicas } & \multicolumn{2}{c}{ ETo estimada_1 } & \multicolumn{2}{c}{ ETo estimada_2 } \\
\cline { 2 - 4 } & \multicolumn{1}{c}{ Índice de concordancia } & Índice de confianza & Índice de concordancia & Índice de confianza \\
\hline \multirow{2}{*}{ Guaro } & 0,8949 & 0,7305 & 0,7984 & 0,5603 \\
Pinares de Mayarí & 0,8618 & 0,6478 & 0,7272 & 0,4916 \\
\hline
\end{tabular}


y Pinares de Mayarí, respectivamente. En el caso de la ETo estimada_2, el índice de confianza se ubicó en las categorías de tolerable y pobre para las estaciones meteorológicas mencionadas, en el mismo orden, anteriormente.

Souza et al. (2015) al evaluar diferentes metodologías de determinación del coeficiente $K p$ utilizados en la corrección de los valores del tanque evaporímetro clase "A" para la estimación de la evapotranspiración de referencia en la región de Sinop, estado de Mato Grosso (Brasil), encontraron mayores índices de concordancia $(d)$ y de desempeño (c) para el coeficiente $K p$ directo que para el determinado a partir de la ecuación de Allen et al. (2006).

También, el comportamiento del coeficiente $K p$ determinado a partir de la ecuación propuesta por Allen et al. (2006) coincide con lo observado por Bonet et al. (2012), quienes encontraron diferencias significativas entre la ETo estimada con los coeficientes $K p$ mencionados anteriormente con respecto a la ETo estimada a partir de los coeficientes obtenidos por la ecuación del tanque evaporímetro clase "A".

\section{Conclusiones}

Los modelos de regresión con mejores coeficientes se obtuvieron en las dos estaciones meteorológicas con la ETo estimada_1 con los coeficientes $K p$ calibrados a partir de la ecuación del tanque evaporímetro clase "A".

La ETo estimada_1 mostró, en ambas estaciones meteorológicas, los índices de concordancia más cercanos a 1. De la misma manera, los índices de confianza de esta ETo se clasificaron como bueno y medio.

La estimación de la evapotranspiración de referencia a partir de la ecuación del tanque evaporímetro clase "A", mediante el uso de los coeficientes $K p$ calibrados por esta misma ecuación, constituye un método óptimo, rápido y sencillo. Por lo que puede constituir una herramienta adecuada para el manejo del riego.

\section{Literatura Citada}

Allen, R.G.; Doorenbos, J.; Monteith, L.

2006. Evapotranspiración del cultivo. Guía para la determinación de los requerimientos de agua de los cultivos. Estudio FAO Riego y Drenaje, boletín 56, Roma. 298 p.

Andrade, A.D.; Miranda, W.L.; Carvalho, L.G. de; Figueiredo, P.H.F.; Silva, T.B.S. da.

2016. Desempenho de métodos de cálculo do coeficiente de tanque para estimativa da evapotranspiração de referencia. Irriga, 21(1): 119-130.

Bonet Pérez, C.; Brown Manrique, O.; Duarte Díaz, Carmen. 2012. Coeficiente de evaporímetro para la programación del riego. Ingeniería Agrícola, 2(1): 26-30.

Camargo, A.P.; Sentelhas, P.C.

1997. Avaliação do desempenho de diferentes métodos de estimativa da evapotranspiração potencial no Estado de São Paulo. Revista Brasileira de Agrometeorologia, 5: 89-97.

Martins de Souza, J.; Rosa Pereira; L.; Matta Rafael, A. da; Dias da Silva, L.; Fialho dos Reis, E.; Bonomo, R.
2014. Comparison of methods for estimating reference evapotranspiration in two locations of Espirito Santo. Revista Brasileira de Agricultura Irrigada, 8(2): 114-126.

Puppo, L.; García Petillo, M. 2009. Estimación de los coeficientes de tanque "A" para calcular la evapotranspiración del cultivo de referencia en la zona sur del Uruguay. Agrociencia, 13(2): 47-51.

Sánchez Martínez, M.I.

2001. Métodos de estimación de evapotranspiración utilizados en Chile. Revista de Geografía Norte Grande, 28: 3-10.

Sentelhas, P.; Folegatti, M.

2003. Class "A" pan coefficients $(K p)$ to estimate daily reference evapotranspiration (ETo). Revista Brasileira de Engenharia Agrícola e Ambiental, 7(1): 111-115.

Souza, A.P.; Almeida, F.T.; Arantes, K.R.; Martim, C.C.; Silva, J.O.

2015. Coeficientes de Tanque Classe A para estimativa da evapotranspiração de referência diária na região de transição Cerrado-Amazônica. Scientia Plena, 11(5): 1-13.

Willmott, C.J.

1985. On the validation of models. Physical Geography. 2: 84-194. 
